Review began 10/25/2021 Review ended 11/08/2021 Published 11/10/2021

๑) Copyright 2021

Songur et al. This is an open access article distributed under the terms of the Creative Commons Attribution License CC-BY 4.0., which permits unrestricted use, distribution, and reproduction in any medium, provided the original author and source are credited.

\section{Evaluation of the Usefulness of YouTube Videos on Retinal Detachment Surgery}

\author{
Murat Serkan Songur $^{1}$, Mehmet Citirik ${ }^{2}$ \\ Research Hospital, Ankara, TUR \\ Corresponding author: Murat Serkan Songur, mssongur@yahoo.com
}

1. Ophthalmology, Bozok University Faculty of Medicine, Yozgat, TUR 2. Ophthalmology, Ulucanlar Eye Education and

\section{Abstract \\ Introduction}

The aim of this study is to evaluate the usefulness of YouTube videos about retinal detachment surgery as a resource.

\section{Methods}

The first 100 videos were evaluated when they were scanned by typing "retinal detachment surgery " in the YouTube search engine. These videos were also analyzed and scored using DISCERN, Journal of the American Medical Association (JAMA), and Global Quality (GO) scoring systems.

\section{Results}

The DISCERN score of the evaluated videos was $39.5 \pm 8.4$; JAMA score was $1.9 \pm 0.5$; and the GQ score was $2.1 \pm 0.5$. According to the results, retinal detachment surgery videos, DISCERN score is medium; The JAMA score was evaluated as low quality and poor quality in the GQ score.

\section{Conclusion}

Although there are enough videos on YouTube with retinal detachment surgery, its usefulness as a resource is low, and its quality is poor.

Categories: Ophthalmology

Keywords: global quality score, jama score, discern score, youtube, retinal detachment surgery

\section{Introduction}

Retinal detachment is the separation of the neurosensory retina (NSR) from the underlying retinal pigment epithelium (RPE) [1]. Although the pathogenesis of retinal detachment is not completely understood, symptomatic retinal tear is often associated with vitreous traction and carries a great risk for retinal detachment [2]. The most common type of retinal detachment is rhegmatogenous retinal detachment. The incidence of retinal detachment is also increasing after cataract surgery [3]. In the treatment of retinal detachment, scleral buckling, pars plana vitrectomy (PPV), pneumatic retinopexy, and combination techniques are used [4]. Air, sulfur hexafluoride (SF6) gas, perfluoropropane (C3F8) gas, and intravitreal silicone tamponade can be used in patients with retinal detachment treated with PPV [5].

YouTube has become one of the most popular websites in the world, with over two billion users and over 500 hours of video content uploaded to the website every minute [6]. The internet has provided the opportunity to access almost all necessary information easily and free of charge. The increasing availability of video sharing sites, such as YouTube, has led to their use as a tool for obtaining information in the field of health as well as in other fields. However, using YouTube as a source of information in the field of health can cause various problems. Problems such as uploading videos by non-healthcare professionals, presentation of opinions without sufficient knowledge and experience, videos used for advertising purposes, lack of detailed information about the contraindications and complications of an operation or procedure, and the lack of a controlling evaluation process occur. In addition, patients and individuals with incomplete medical knowledge may evaluate health-related information from their own perspective, which may lead to the dissemination of incomplete or inaccurate information [7].

Our aim in this study is to evaluate retinal detachment surgery videos that have not been evaluated before in the literature and to provide an overview by evaluating the quality and educational contribution of these videos through scoring.

\section{Materials And Methods}




\section{Cureus}

This study was conducted in September 2021 by retrospectively viewing videos that were publicly available on YouTube. The principles of the Declaration of Helsinki were adhered to during the study. We evaluated the first 100 videos by typing "retinal detachment surgery" in the YouTube search engine. These videos were recorded in terms of duration, likes and dislikes, release date, content, and the number of views. Only videos in English were taken into consideration.

The videos were independently evaluated by the two authors, who are experienced ophthalmologists. All videos were independently scored by DISCERN, Journal of the American Medical Association (JAMA), and Global Quality (GQ), and the results were averaged.

There are 16 questions in total in DISCERN scoring. The score of all questions ranges from one to five. The first eight questions are used to determine the credibility of the web page. The second part, 9 through 15, evaluates the quality of information about treatment options. The 16th and final question is a general evaluation of the website. There is a score between 16 and 75 in DISCERN scoring. In DISCERN scoring, it is classified as very poor between 16 and 26 points, poor between 27 and 38, moderate between 39 and 50 , good between 51 and 62, and excellent between 63 and 75 [8]. DISCERN questions are shown in Table 1.

Questions in the DISCERN scoring system

\section{SECTION 1}

Question 1

Question 2

Question 3

Question 4

Question 5

Question 6

Question 7

Question 8

SECTION 2

Question 9

Question 10

Question 11

Question 12

Question 13

Question 14

Question 15

SECTION 3

Question 16

Global Quality (GQ) scoring system

1 - Bad quality

2 - Bad quality

3 - Insufficient quality and flow

4 - Good quality and streaming

5 - Excellent quality and flow
Are the goals clear?

Does it reach its goals?

Is it relevant?

Are the publication sources used to compile information compatible?

Is it clear when information is used or reported?

Is it balanced and unbiased?

Does it provide additional support resources and information?

Does it refer to indefinite fields?

Does it explain how each treatment works?

Does it explain the benefits of each treatment?

Does it explain the risks of each treatment?

Does it explain what can happen if left untreated?

Does it explain how much each treatment can affect quality of life?

Does it explain that there may be more than one possible treatment choice?

Does it provide support for joint decision making?

What is the overall quality rating like?

Not likely to be used for patient education

Limited use for patients; because only some information is available

It is somewhat helpful; important topics are missing, some information is available

It is useful for patients; because the most important topics are covered

Very useful for patients

TABLE 1: Questions in the DISCERN scoring system and the Global Quality (GQ) scoring system 


\section{Cureus}

JAMA criteria are used to evaluate basic information presented on websites. Basically, it includes authorship, bibliography, patent right, and timeliness. Each criterion gets one point. A one indicates the weakest quality and a four indicates the highest quality [9].

GQ scoring provides the opportunity to interpret the videos in general and to evaluate the overall quality of the videos according to the information flow presented [10]. In the GQ scoring, the scoring ranges from one to five. The GQ scoring system is shown in Table 1 [11].

All analyzes were performed using the SPSS Windows V.21.0 software package (IBM Inc., Armonk, New York). The mean SD was used for continuous data. Student T-test was used for pairwise comparison of data for normally distributed data. Spearman correlation test was applied to examine the relationships between the variables. A p-value less than 0.05 was considered significant.

\section{Results}

In our study, 87 videos that met the inclusion criteria out of a total of 100 videos that emerged as a result of searching for the keyword in the YouTube search engine were analyzed and evaluated. Of these videos, three were excluded because they were irrelevant, three were in a language other than English, and seven were excluded because of the content of retinal diseases and surgery other than retinal detachment surgery. The general features of the videos are summarized in Table 2.

\begin{tabular}{|c|c|c|c|}
\hline \multicolumn{4}{|c|}{ General features of videos } \\
\hline \multicolumn{2}{|c|}{ Broadcast time (seconds) } & \multicolumn{2}{|l|}{805.5} \\
\hline \multicolumn{2}{|l|}{ Number of likes } & \multicolumn{2}{|l|}{169.5} \\
\hline \multicolumn{2}{|l|}{ Number of dislikes } & \multicolumn{2}{|l|}{7.5} \\
\hline \multicolumn{2}{|c|}{ Broadcast history (month) } & \multicolumn{2}{|l|}{39.2} \\
\hline \multicolumn{2}{|l|}{ Views } & \multicolumn{2}{|l|}{27114.5} \\
\hline \multicolumn{4}{|c|}{ Comparison of videos with and without surgery } \\
\hline & Videos containing surgery $(n=58)$ & Non-surgical videos $(n=26)$ & $p$-value \\
\hline Video duration (sec) & $874.5 \pm 125.6$ & $728.2 \pm 168.6$ & 0.646 \\
\hline Number of likes & $64.2 \pm 14.4$ & $287.7 \pm 50.0$ & 0.005 \\
\hline Number of dislikes & $3.7 \pm 1.2$ & $11.7 \pm 2.1$ & 0.036 \\
\hline Views & $12689.0 \pm 449.1$ & $43299.3 \pm 618.5$ & 0.009 \\
\hline DISCERN score & $41.5 \pm 5.6$ & $37.2 \pm 10.3$ & 0.016 \\
\hline JAMA score & $2.1 \pm 0.4$ & $1.7 \pm 0.5$ & 0.001 \\
\hline Global Quality score & $2.2 \pm 0.4$ & $1.9 \pm 0.6$ & 0.008 \\
\hline
\end{tabular}

\section{TABLE 2: General features of videos and comparison of videos with and without surgery}

None of the evaluated videos contained advertising content. All of the videos were uploaded by ophthalmologists. There was a significant correlation between the viewing rates of all videos and the number of likes $(\mathrm{p}<0.001 ; \mathrm{r}=0.850)$ and dislikes $(\mathrm{p}=<0.001 ; \mathrm{r}=0.817)$. No correlation was found between the duration of the videos and the rate of viewing ( $\mathrm{p}=0.978 ; \mathrm{r}=0.273$ ). In the videos, 46 videos were surgical, while 41 videos did not have a surgical presentation. There was no statistically significant difference between the duration of the videos with and without surgery. The number of likes and dislikes and the number of views of the non-surgical videos were statistically significantly higher than the surgical videos. However, videos with surgical content were found to have statistically significantly higher quality in DISCERN ( $p=0.016)$, JAMA $(p=0.001)$, and $\mathrm{GQ}(\mathrm{p}=0.008)$ scores than videos without surgical content (Table 2).

The DISCERN score of the evaluated videos was $39.5 \pm 8.4$; JAMA score was $1.9 \pm 0.5$; and the GQ score was $2.1 \pm 0.5$. According to the results, retinal detachment surgery videos, DISCERN score was moderate; The JAMA score was assessed as low quality and the GQ scoring as poor quality. 


\section{Discussion}

In our study, we found that in videos on retinal detachment surgery on YouTube, the DISCERN score was moderate, the JAMA and GQ scores were low, and the videos were of insufficient quality. We also found that all three scores were higher in videos with surgical content than those without surgical content.

YouTube is the world's largest media-sharing website and the second most used website worldwide. In addition to providing a platform for information and moral support, the internet has created opportunities for open discussion about health and medicine. Unfortunately, this increased opportunity has led to the increase and dissemination of false and even harmful information. The rise in published studies on YouTube video reliability is proof of this [12].

Borgersen et al., in their study, determined that only 27 of 7640 videos were suitable for use in the YouTube video search for direct ophthalmoscope [13]. Benetoli et al. revealed that many social media users turn to health-related YouTube videos for emotional support, especially during a chronic illness. However, they stated that since many people do not trust these videos, they still want to meet with doctors face-to-face for information [14]. Sahin et al. found that one-third of the videos on retinopathy of prematurity on YouTube were misleading and could have harmful consequences [15]. Abdelmseih et al. examined the quality of YouTube videos in age-related macular degeneration. Of the videos they reviewed, $60 \%$ were rated as partially applicable, $35 \%$ as misleading, and $5 \%$ as irrelevant. According to reliability, $60 \%$ of videos are classified as partially reliable, $35 \%$ as unreliable, and only $5 \%$ as reliable [16].

Aykut et al., in their study where they examined YouTube videos of cataract surgeries performed on eyes with small pupils, found that only one of the videos had significant complications and one had minor complications. However, considering that the complication rate is normally higher in eyes with small pupils, concluded that such videos are published less frequently [17]. Similarly, no complications were found in any of the videos in our study.

Nicholl et al. reported that parents preferred accurate information (65\%), reliable information (62\%), and updated information (61\%) in information research [18]. Since this shows especially DISCERN and JAMA scoring, we think that it may be appropriate to use these scorings in video evaluation. In their YouTube study of contact lenses, Yildiz et al. found the videos have an average DISCERN score of videos to be 32.3, JAMA score to be 1.2, and GQ score to be 3.4. They stated that the video quality is higher in videos published by universities and professional organizations [19]. In Küçük et al.'s review of refractive surgery videos on YouTube, the average DISCERN score of the videos was 33.2; JAMA score of 0.7 ; they found that the GQ score was 1.7 and as a result, the video quality was poor [20]. In our study, the mean DISCERN score was 39.5; The JAMA score was 1.9, and the GQ score was 2.1 and the videos were generally of poor quality. As far as we know, there is no scientific article examining retinal detachment surgery videos on YouTube.

Online videos for patient education and the issues with their quality and accuracy have received more attention recently. Social media has great potential to provide easy access to medical information, but it is not possible to ensure that the information received is accurate and unbiased.

Our study has several limitations. First of all, we do not have enough information about the pre- and postoperative videos that we consider surgical. Secondly, we evaluated videos in English. Third, the video evaluation was subjective, although it was evaluated independently by two experienced surgeons. Further studies are needed to evaluate youtube videos in retinal detachment.

As a result of this study, YouTube videos labeled "retinal detachment surgery" often contain poor content quality and incomplete information. In order for these videos to be used as a source of information, they should be recorded by more qualified professionals, presenting their content and all information about all treatment options, complications, and healing processes in an objective way.

\section{Conclusions}

As a result, according to our findings, YouTube videos labeled "retinal detachment surgery" often contain poor content quality and incomplete information. In order for these videos to be used as a source of information, they should be recorded by more qualified professionals, presenting their content and all information about all treatment options, complications, and healing processes in an objective way.

\section{Additional Information \\ Disclosures}

Human subjects: All authors have confirmed that this study did not involve human participants or tissue. Animal subjects: All authors have confirmed that this study did not involve animal subjects or tissue. Conflicts of interest: In compliance with the ICMJE uniform disclosure form, all authors declare the following: Payment/services info: All authors have declared that no financial support was received from any organization for the submitted work. Financial relationships: All authors have declared that they have 
no financial relationships at present or within the previous three years with any organizations that might have an interest in the submitted work. Other relationships: All authors have declared that there are no other relationships or activities that could appear to have influenced the submitted work.

\section{References}

1. Steinberg RH, Wood I: Pigment epithelial cell ensheathment of cone outer segments in the retina of the domestic cat. Proc R Soc Lond B Biol Sci. 1974, 187:461-78. 10.1098/rspb.1974.0088

2. Williams KM, Dogramaci M, Williamson TH: Retrospective study of rhegmatogenous retinal detachments secondary to round retinal holes. Eur J Ophthalmol. 2012, 22:635-40. 10.5301/ejo.5000080

3. Ramos M, Kruger EF, Lashkari K: Biostatistical analysis of pseudophakic and aphakic retinal detachments . Semin Ophthalmol. 2002, 17:206-13. 10.1076/soph.17.3.206.14784

4. Schwartz SG, Flynn HW: Primary retinal detachment: scleral buckle or pars plana vitrectomy? . Curr Opin Ophthalmol. 2006, 17:245-50. 10.1097/01.icu.0000193097.28798.fc

5. Tan HS, Oberstein SY, Mura M, Bijl HM: Air versus gas tamponade in retinal detachment surgery . Br J Ophthalmol. 2013, 97:80-2. 10.1136/bjophthalmol-2012-302140

6. YouTube statistics. (2021). Accessed: 2021: https://backlinko.com/youtube-users\#youtube-statistics.

7. Kocyigit BF, Akaltun MS: Does YouTube provide high quality information? Assessment of secukinumab videos. Rheumatol Int. 2019, 39:1263-8. 10.1007/s00296-019-04322-8

8. Singh AG, Singh S, Singh PP: YouTube for information on rheumatoid arthritis--a wakeup call? . J Rheumatol. 2012, 39:899-903. 10.3899/jrheum.111114

9. Silberg WM, Lundberg GD, Musacchio RA: Assessing, controlling, and assuring the quality of medical information on the Internet: caveant lector et viewor--let the reader and viewer beware. Generations. 1997, 21:53-5.

10. Weil AG, Bojanowski MW, Jamart J, Gustin T, Lévêque M: Evaluation of the quality of information on the Internet available to patients undergoing cervical spine surgery. World Neurosurg. 2014, 82:e31-9. 10.1016/j.wneu.2012.11.003

11. Erdem MN, Karaca S: Evaluating the accuracy and quality of the information in kyphosis videos shared on YouTube. Spine (Phila Pa 1976). 2018, 43:E1334-9. 10.1097/BRS.0000000000002691

12. Moorhead SA, Hazlett DE, Harrison L, Carroll JK, Irwin A, Hoving C: A new dimension of health care: systematic review of the uses, benefits, and limitations of social media for health communication. J Med Internet Res. 2013, 15:e85. 10.2196/jmir.1933

13. Borgersen NJ, Henriksen MJ, Konge L, Sørensen TL, Thomsen AS, Subhi Y: Direct ophthalmoscopy on YouTube: analysis of instructional YouTube videos' content and approach to visualization. Clin Ophthalmol. 2016, 10:1535-41. 10.2147/OPTH.S111648

14. Benetoli A, Chen TF, Aslani P: Consumer health-related activities on social media: exploratory study . J Med Internet Res. 2017, 19:e352. 10.2196/jmir.7656

15. Şahin A, Şahin M, Türkcü FM: YouTube as a source of information in retinopathy of prematurity . Ir J Med Sci. 2019, 188:613-7. 10.1007/s11845-018-1902-2

16. Abdelmseih M: Evaluation and reliability of YouTube videos for age-related macular degeneration (AMD) - a warning sign!. Clin Experiment Ophthalmol. 2016, 7:10.4172/2155-9570.1000595

17. Aykut A, Kukner AS, Karasu B, Palancıglu Y, Atmaca F, Aydogan T: Everything is ok on YouTube! Quality assessment of YouTube videos on the topic of phacoemulsification in eyes with small pupil. Int Ophthalmol. 2019, 39:385-91. 10.1007/s10792-018-0823-4

18. Nicholl H, Tracey C, Begley T, King C, Lynch AM: Internet use by parents of children with rare conditions: findings from a study on parents' web information needs. J Med Internet Res. 2017, 19:e51. 10.2196/jmir.5834

19. Yildiz MB, Yildiz E, Balci S, Özçelik Köse A: Evaluation of the quality, reliability, and educational content of YouTube videos as an information source for soft contact lenses. Eye Contact Lens. 2021, 47:617-21. 10.1097/ICL.0000000000000795

20. Kuçuk B, Sirakaya E: An analysis of YouTube videos as educational resources for patients about refractive surgery. Cornea. 2020, 39:491-4. 10.1097/ICO.0000000000002237 